\title{
La gestión de recursos humanos, enfoques y perpectivas
}

Fecha de recepción: 18 de marzo de 2010

Fecha de aprobación: 1 junio de 2010

\author{
Aura Janeth Uribe Arévalo \\ uribea@hotmail.com \\ Fundación Universitaria Empresarial de la Cámara de Comercio de Bogotá
}

\section{Reseña del autor}

Profesional en Administración de Empresas, magíster en Educación, candidata al doctorado Nuevas Tendencias en Dirección de Empresas en la Universidad de Valladolid; docente-investigador de la Fundación Universitaria Empresarial de la Cámara de Comercio de Bogotá.

\section{Resumen}

La modernidad trae el desarrollo tecnológico y mejores medios para desarrollar procesos, pero el hombre y su capacidad seguirán siendo insustituibles. Una óptima gestión de recursos humanos genera conocimiento, maximización y creación de recursos, y un personal dispuesto a crecer con la empresa.

La gestión de recursos humanos es un campo de estudio e investigación considerado desde la economía, la estrategia y la gestión. Desde una óptica humanista y de producción, la participación del ser humano en las organizaciones es importante; en el sentido de conocer la empresa como una gestora de relaciones, de contratos y administradora de recursos; y también preocupante en términos de regulación, administración y control, desde el momento de su vinculación a través de las relaciones contractuales.

\begin{abstract}
Modernity brings technological development and better ways to develop proceedings, but the man and his ability will be remain irreplaceable. Optimum management of human resources produces knowledge, resources maximizing and building, and a staff willing to grow with the company.

Human resources management is a field of study and research seen from economics, strategy, and management. From a humanist perspective and production, participation in organizations is important; in the sense of knowing the firm as a managing relationships, contracts and resources administrator; but disturbing in terms of regulation, administration and control, from the moment of their relationship through contractual relationships.

This paper wants to highlight the importance of human resources in companies
\end{abstract}


Este documento resalta la importancia de los recursos humanos en las empresas y sus beneficios para potencializar y hacer más competitivas a las organizaciones actuales.

El artículo se construye desde la revisión teórica de tres conceptos básicos para la gestión de recursos humanos: el contractual, el de recursos y capacidades y el de conocimiento. Los tres apartados recogen las consideraciones de cada enfoque, su impacto en la realidad empresarial y las tendencias sobre futuras investigaciones.

Estos enfoques, en particular, fueron escogidos por la tendencia de las investigaciones más recientes y su vinculación con el rendimiento de la empresa.

\section{Palabras clave}

Teoría de agencia, gestión, recursos humanos, gestión del conocimiento.

\section{El enfoque contractual}

Los aportes realizados por Jensen y Meckling (1976) definen una relación de agencia como "un contrato en el que una o más personas -el principal/escontratan a otra persona-el agente- para realizar un determinado servicio, lo que implica cierto grado de delegación de autoridad en el agente"(p. 308). De esta forma, el agente principal tratará de eliminar o desviar los intereses personales del agente ofreciéndole incentivos o creando mecanismos de control para supervisar su gestión. Aunque resulte difícil optimizar la relación de agencia sin incurrir en costos, la asimetría de and their benefits to empower and make it more competitive with the actual organizations.

The article is constructed from the theoretical review of three basic concepts for human resource management: the contract, the resources and capabilities and knowledge. The three headlands set out its considerations of each approach, its impact on business realities and future research trends.

These approaches, in particular, were chosen by the tendency of recent research and its role in a company's performance.

Key words

Agency Theory, Management, Human Resources, Knowledge Management. información será prácticamente imposible de vencer.

La relación de agencia puede darse en varios niveles y en diferentes tipos de organización. Desde esta perspectiva, se define el enfoque contractual para los recursos humanos, se analizan los diversos contratos de la empresa y se determinan sus relaciones e implicaciones. Los postulados de la teoría de agencia pueden aplicarse a diversas relaciones de agentes económicos, y trasladarse a relaciones contractuales generadas en la empresa, es decir, relaciones de empleo (Pérez, 1999). 
Pérez (1999) expone los tipos de contrato que surgen en la empresa:

- Explícitos o implícitos, según el grado de formalización.

- De largo o corto plazo, según el tiempo.

- Completos e incompletos, según la especificidad del tipo de contrato y su grado de dificultad.

- Multilaterales o bilaterales, según el número de participantes.

- De control, según del comportamiento de acuerdo a los resultados.

La teoría de agencia muestra los mecanismos para disciplinar al directivo cuando surgen los conflictos. Para el caso del enfoque contractual de recursos humanos, los conflictos se presentan entre el empleado y el empleador, debido a la incertidumbre y a la desigualdad de la información (Pérez, 1999).

La retribución es uno de los mecanismos de mayor utilización para lograr la disciplina. Una política retributiva adecuada puede minimizar los riegos en los contratos y lograr que el empleado cumpla con sus obligaciones. Las empresas requieren de una política retributiva clara que se nutra de investigaciones del mercado externo, de las capacidades de sus colaboradores y de las tendencias, para buscar, ante todo, un pago justo.

El impacto tecnológico en los procesos de producción, unido a la creciente globalización de los mercados, ha obligado a las empresas a reinventar continuamente sus productos y servicios, y a diferenciarse a través de sus intangibles. Esta búsqueda supone un diseño más efectivo de las capacidades de la empresa, que obliga a alterar los procesos organizativos y estratégicos para adaptarse al dinamismo del mercado.

Durante el siglo xxI han surgido nuevas empresas con nuevas formas de constitución, que trascienden el modelo clásico de empresa y recurren a modelos más flexibles, considerados una nueva forma de estructuración organizacional, con variaciones en los pesos de los costos de control -y/o de coordinación- y de motivación -y/o de incentivos-. Se hace mayor énfasis en éstos, ya que recaen directamente en la gestión de recursos humanos.

Desde la perspectiva contractual, los mecanismos de control y motivación buscan evitar el oportunismo contractual en el ámbito laboral. Existen tres clases de problemas de oportunismo:

1. De compromiso: surgen como consecuencia de contingencias no especificadas de antemano en los contratos.

2. De riesgo moral: surgen de la dificultad de observación y medición de la contribución de cada individuo a la acción colectiva.

3. De selección adversa: surgen como consecuencia del oportunismo precontractual de los individuos.

Las nuevas empresas buscan la reducción de los oportunismos mediante 
la aplicación de técnicas organizativas, lo que supone una estructura de bajo costo que abarate el funcionamiento de unidades de la empresa, y mediante la revisión de los contratos que afecten las relaciones laborales.

Las empresas se encuentran en procesos de ajuste y dinámicas de reconstrucción de sus procesos internos, estratégicos y prospectivos. De igual manera, han despertado interés en capacitarse con el fin de cautivar nuevos profesionales comprometidos con los objetivos de la empresa, por lo que se redefine la dirección de recursos humanos desde una óptica de gestión del talento.

En la actualidad, los recursos humanos de la empresa son la clave de su gestión. El conocimiento, capaz de generar desarrollo e innovación, explica el surgimiento de nuevas formas de relaciones para estos nuevos profesionales. Desde esta nueva realidad empresarial, resulta necesario ahondar en el estudio de las relaciones contractuales en las nuevas empresas, a través de diversas perspectivas de investigación.

- Estudio de las transacciones laborales se refiere a las relaciones que suponen contratos incompletos y que determinan la eficiencia de las empresas y a las de la competitividad, determinadas por las brechas en los intereses entre empleado y empleador que, sumadas a las diferencias en la información, requieren de la coordinación y del control de incentivos para corregir los conflictos.

- Construcción de activos estratégicos de recursos humanos y el diseño de estrategias de retención: son mecanismos de remuneración, seguridad en el empleo, formación y desarrollo de carreras, que fomentan un sentimiento de pertenencia hacia la organización.

- Visión estratégica de los recursos humanos de la empresa, que se proyecte con la capacidad de generar y transferir la dinámica del conocimiento de sus recursos humanos, es decir, que los conocimientos individuales generen un conocimiento organizacional.

Se requiere tener una visión más amplia del personal y articularse con la gestión de conocimiento, teniendo en cuenta la movilidad, la geoespacialidad de los recursos humanos y su transitoriedad. Las empresas son cada vez más flexibles y deben considerar empleados flexibles. Desde el enfoque contractual se debe anticipar las formas de maximizar las nuevas relaciones que garanticen un flujo en la comunicación y una participación de toda la organización.

Asimismo, se deben definir cuáles son los estilos de dirección apropiados para los cambios organizacionales, en los que las empresas del siglo xxi están inmersas; y considerar si los estilos de dirección tradicionales son los 
apropiados, o si ya se puede hablar de una nueva forma de dirigir a los recursos humanos de las nuevas empresas.

Actualmente, las empresas viven en un mundo globalizado, que ofrece ambientes de multiculturalidad y diversidad étnica, lo que obliga a investigar cómo son las nuevas relaciones en estas empresas y su dirección, comunicación e incentivos.

\section{Enfoque de recursos y capacidades}

La teoría de recursos y capacidades procede del ámbito académico -se desarrolla en los años 80 - y se considera la antecesora de la gestión del conocimiento -años 90-. Consiste en trascender a una visión de empresa basada en recursos, para luego aproximarse a lo que debe ser una dirección estratégica de recursos humanos, lo que supone la existencia de un compromiso de los empleados. La dirección de recursos humanos considera al personal como un elemento para ser optimizado (Saa y García, 2000).

Por otra parte, la teoría busca explicar por qué dentro del mismo sector económico se producen diferencias en el desempeño de las organizaciones, por qué el estudio de la estrategia avanzó hacia el paradigma de la estrategia basada en los recursos y capacidades que se poseen -enfoque interno- o los recursos que se deben adquirir para poder competir.

Se trata de ampliar la visión de las organizaciones desde un enfoque exterior a uno interior, en busca de la creación de ventajas competitivas, generadas a partir de los activos estratégicos (Amit y Belcourt, 1999). Las estrategias son una fuente importante para este fin y deben de superar la visión de coste a la empresa (Saa y García, 2000).

La rentabilidad de la empresa se explicaba desde la mirada de atractivo del sector, el grupo estratégico y el crecimiento del mercado, lo que suponía que las empresas pertenecientes a un mismo sector eran iguales en cuanto a recursos y estrategias disponibles. Los estudios recientes expresan diferencias en los resultados de las empresas que se mantienen a lo largo del tiempo, y ante esto, se preguntan si todas las empresas de un mismo sector o grupo estratégico tienen las mismas oportunidades, afirmación que explicaría las diferencias en los resultados. Al relacionar la gerencia de recursos humanos con los resultados de la empresa, es posible obtener valores adicionales en cuanto al capital humano y se logran mejores prácticas de gestión (Amit y Belcourt, 1999).

En Saa y García (2000) se encuentra que la empresa puede alcanzar rentas superiores como la consecuencia de un mejor uso de sus recursos. La función del estratega es encontrar un adecuado equilibrio entre las oportunidades y las 
amenazas del entorno, con las fortalezas y debilidades de la empresa, basado en la existencia de las competencias distintivas para competir con éxito, concepto planteado por la teoría de los recursos e impulsada por dos hechos:

1. Las diferencias de resultados intersectoriales son menores que las diferencias intrasectoriales.

2. Los aspectos internos de la empresa ofrecen factores explicativos de sus resultados.

En esta teoría se concibe la empresa así:

1. Se abandona la idea de empresa como caja negra, para considerarla como un elemento más, inmerso en una teoría de precios y mercados más amplia, que puede contribuir significativamente a explicar diferentes aspectos.

2. Se asume que existen diferencias entre empresas y se descarta el concepto clásico de que todas las empresas son iguales en términos de los recursos controlados y los productos ofrecidos. Amit \& Belcourt (1999) plantean en su artículo la heterogeneidad de los recursos y capacidades de las empresas.

En otras palabras, la empresa se concibe como una institución compleja que nace con el propósito de mediar y colaborar entre los propietarios de los recursos. Posee recursos como el know-how de los empleados, la reputación, las marcas y la habilidad para trabajar en equipo. El capital humano es visto como un activo estratégico, aunque no como una posesión de la empresa, por tratarse de un intangible (Amit \& Belcourt, 1999).

Se ha considerado que el recurso más importante en la empresa es el humano, estudiado como una herramienta valiosa en los términos definidos por esta teoría. La valoración de un modelo o de las recomendaciones encaminadas a la obtención de ventajas competitivas para la empresa se logra mediante la aplicación de indicadores de gestión de recursos humanos, que miden los efectos sobre la productividad. De este modo se consigue una ventaja competitiva sobre la base del desarrollo de un compromiso de los empleados con la empresa.

La teoría de los recursos define la empresa como una unidad poseedora de recursos y capacidades única y la teoría de recursos y capacidades la concibe con el nivel de análisis adecuado y su misión principal es el estudio de las diferencias en los resultados empresariales.

La heterogeneidad entre empresas es el factor que fortalece la teoría de los recursos, se mantiene a lo largo del tiempo y se debe a factores internos y externos. Uno de los postulados fundamentales de esta teoría consiste en que los resultados de la empresa dependen de la explotación del potencial de los recursos y de las capacidades de la empresa para la generación de rentas. 
Al respecto existen las siguientesperspectivas de investigación:

- La identificación de los recursos y capacidades para aspectos sociales, un desafío en el caso de recursos socialmente complejos.

- La construcción de modelos para el desarrollo de recursos y capacidades valiosos.

- El análisis de los competidores, indispensable al formular la estrategia competitiva de la empresa, pues determina sus puntos fuertes y débiles.

Uno de los conflictos con los recursos es la posibilidad de ser sustituibles, característica que ha recibido poca atención en las investigaciones, dado que puede tratarse de una amenaza superior frente a recursos susceptibles de imitación. Dicha amenaza se materializa en el carácter obsoleto que pueden adoptar los recursos y capacidades de la empresa, si algún competidor desarrolla un sustituto con características superiores al original. Esto podría evitarse mediante una actitud innovadora, que establezca una visión de la teoría de los recursos e integre una visión estática y dinámica -la razón de ser de la empresa y el crecimiento que se logre con la formación-, la explotación de los recursos que posee y el desarrollo de nuevos recursos a partir de los existentes, proceso conocido como innovación.

\section{Enfoque de conocimiento}

Este es un siglo de cambios deseados o buscados, y en esta ambigüedad se encuentran las organizaciones que buscan transformarse mediante los nuevos enfoques administrativos. El valor reside en las personas, en quienes la información y el conocimiento generan una nueva sociedad en el mundo de hoy. El nuevo conocimiento se crea al interior de la organización a través del intercambio de los empleados (Collins \& Smith, 2006). Vera \& Crossan (2003) presentan este aspecto como la gestión de conocimiento, que conduce a cambios en las prácticas de recursos humanos y de gerencia, lo que se traduce en la innovación y en la intersección de conocimiento y personas.

En el mundo contemporáneo, la gente aprende a diversificar su conocimiento y experiencia para fortalecer sus habilidades y su creatividad; de esta manera, aumenta su capacidad para comprender los cambios y accede a las organizaciones, un sector dinámico y cambiante. Según Crossan et al (1999), el aprendizaje organizacional ha existido desde 1965; los autores presentan un proceso de aprendizaje organizacional, llamado el de las $4 I$, que involucra procesos de intuición, que parten del individuo; interpretación, definida como la explicación de ideas a terceros; integración, o desarrollo de entendimiento entre individuos; e institucionalización, entendida como 
la existencia de rutinas de acciones, por ejemplo, en la organización.

La gente experimenta un proceso -un nuevo marco de actualización- que proviene del desarrollo tecnológico, sustentado en la comunicación y en los nuevos modelos gerenciales. La tecnología de la información se considera sólo una herramienta para comunicación (Robertson \& O’Malley, 2000), y los principios básicos de las nuevas formas de dirección consisten en aprender a conocer, a hacer, a convivir con los demás y a establecer principios con un nuevo significado en la gestión del conocimiento, como nuevo paradigma del siglo XxI.

Otra forma de generar nuevo conocimiento es mediante la destrucción creativa, que consiste en dejar atrás órdenes institucionalizadas para permitir que fluyan nuevas rutinas, se inicie la intuición y así se dé paso al proceso de las 4I (Crossan, et al; 1999). Robertson \& O’Malley (2000) muestran cómo la gestión del conocimiento tiende a enfocarse en aspectos de recursos humanos, que se pueden categorizar de la siguiente manera:

- Innovaciones estructurales en el interior las organizaciones, para facilitar la gestión de conocimiento y la dirección del personal.

- La inapropiada aplicación de las prácticas tradicionales de dirección de recursos humanos, que consiste en enfrentar a los trabajadores con conocimiento.
La difusión del conocimiento de aspectos centrales como la calidad, por ejemplo, inicia con la estandarización del conocimiento, más que con una creación del mismo (Robertson, \& O'Malley; 2000). La gestión del conocimiento, por su parte, debe buscar un modelo gerencial que facilite la introducción de nuevas formas de gestión de recursos humanos, la promoción de la adaptabilidad, el autocontrol, la confiabilidad, la iniciativa y el desarrollo del personal. Asimismo, debe apoyarse en las nuevas tecnologías, para estar acorde con las tendencias de la nueva economía. De esta manera, los recursos humanos se adaptan a las exigencias de la nueva visión sobre un concepto de trabajo diferente.

La creación de valor de conocimiento sobre los bienes o servicios ha logrado un recorrido hacia la generación de la rentabilidad organizacional y una estrategia de crecimiento económico, especialmente en organizaciones que invierten en tecnología, en formación de formadores y en el fomento de líderes en nuevos procesos. Para este modelo se proponen los siguientes pasos, principios básicos de una organización que busca la competitividad y orientada hacia gestión del conocimiento:

- Crear, delimitar, compartir y poner en práctica una visión compartida y de equipo.

- Evaluar la cultura organizacional.

- Analizar la capacidad de aprendizaje de la organización. 
- Eliminar las barreras que obstaculizan el cambio.

- Compartir y discernir lo aprendido.

La gestión del conocimiento es vista como un esfuerzo de las organizaciones por conseguir, organizar, distribuir y compartir la información entre todos empleados. Esta nueva práctica transforma la concepción de los modelos tradicionales, ya que su reto es insertarse en el entorno competitivo global, y sobrevivir a través de la implementación de este modelo alternativo, en el que el sentido piramidal es sustituido por el modelo horizontal. Para esto es necesario comprender que la gestión del conocimiento abarca, como condición interna imprescindible, la gestión de la información, que debe ser una herramienta para la organización, sus grupos $\mathrm{y}$ las personas que los conforman.

Una organización inteligente promueve el aprendizaje, la socialización y la incorporación de nuevos conocimientos, con el fin de mejorar y motivar capacidades creativas en las personas; también plantea la integralidad de objetivos, a través de la generación de conciencia y del trabajo en equipo. Con esta percepción, los recursos humanos se encuentran en el centro del escenario, pues son su principal activo. Por esto, la comunicación cobra importancia, para que el conjunto de información corporativa y los conocimientos sociales, organizacionales e individuales sean asequibles.
En muchos casos, la introducción de la gestión del conocimiento se suspende, porque las empresas no la consideran un requisito previo ni una herramienta eficiente y efectiva de gestión de información y comunicación. Sin embargo, la información constituye un elemento central para la generación de conocimiento, cuya difusión en la organización requiere la existencia de comunicación, así como garantías de un conocimiento compartido en toda la empresa, como base para el desarrollo constante de la inteligencia y de la innovación.

Existe una relación directa entre la capacidad de aprendizaje de la organización, el concepto y la práctica de la comunicación. Las empresas que favorecen la innovación integran grupos dentro y fuera de la empresa, mediante redes de participación y el fomento de gestión de conocimiento, un medio interactivo dirigido por el mismo conocimiento y servido por redes (Vera \& Crossan, 2003).

En este caso, las perspectivas de investigación son:

- La gerencia de recursos humanos, que tiene un papel crucial: participar, cada vez más activamente, en el ámbito estratégico de la organización, aunque implique un cambio profundo y exija compromisos de todos sus miembros.

- La ruptura con viejas prácticas gerenciales, a partir de la implementación de la gestión del conocimiento, en la que se evidencia un nuevo 
estilo de dirección y el surgimiento del conocimiento.

- Las estrategias de los directivos para transformar, agregar valor y también fomentar una nueva cultura organizacional, a partir de la gestión de conocimiento.

- El reconocimiento de los mecanismos que detienen el flujo de procesos de aprendizaje (Crossan et al; 1999).

A partir del uso de la información y el conocimiento, es posible determinar cuáles son los aspectos y las condiciones idóneas en tecnologías, estructuras y recursos, para iniciar un cambio en la organización. También, es fundamental pronosticar qué pasará en las organizaciones que se planteen la gestión de conocimiento como una forma de ser y la integren en sus valores, cómo serán las organizaciones que innovan en forma continua, cómo se desarrolla la implantación de la gestión del conocimiento y cuáles son los modelos de dirección más apropiados que favorezcan la gestión de conocimiento.

\section{Conclusiones}

El contrato es el mecanismo de control -relación contractual-, utilizado por las empresas para establecer las reglas del juego; y requieren transparencia y una estrategia adecuada para alcanzar los objetivos organizacionales y evitar conflictos.
La productividad de la empresa está relacionada con la competitividad de sus recursos humanos. Para identificar dicha relación, se deben diseñar indicadores de gestión más amplios que midan los efectos de este recurso y su compromiso sobre la productividad.

La dirección del recurso humano debe encaminarse hacia modelos dinámicos e innovadores, que permitan la comunicación y renovación en la organización, que vayan más allá de las prácticas tradicionales, y en los que prime el conocimiento individual y colectivo.

Los estudios se orientan en of recer mayor flexibilidad y carácter dinámico a la medición del recurso humano, pues consideran que éste implica aspectos sociales, también procuran evitar la estandarización con otros recursos.

Como conclusión general, se observa que los enfoques revisados buscan, desde su perspectiva, incrementar la competitividad, productividad y participación del recurso humano en la rentabilidad de la empresa.

La investigación deja en evidencia que desde estos tres enfoques se plantean instrumentos para dinamizar y modernizar la gestión de recursos humanos en las organizaciones. También deja al descubierto la necesidad de crear grupos de investigación que formulen herramientas y mecanismos de evaluación del recurso humano más dinámicos, en concordancia con las necesidades de la empresa y orientados a 
la productividad, para hacer frente a las tendencias de la economía y las nuevas dinámicas organizacionales.

\section{Referencias}

1. Amit, R. \& Belcourt, M. (1999). Human Resources Management Process: a Value-Creating Source of Competitive Advantage. European Management Journal, No. 17 (2), pp. 174-181.

2. Collins, C.J. \& Smith, K.G. (2006). Knowledge Exchange and Combinations: the Role of $\mathrm{Hu}^{-}$ man Resource Practices in the Performance of High-Technology Firms. Academy of Management Journal, No. 49 (3), pp. 544-560.

3. Crossan, M.M.; Lane, H.W. \& White, R.E. (1999). An organizational Learning Framework: from Intuition to institution. Academy of Management Review, No. 24 (3), pp. 522-537.

4. Jensen, M.C. \& Meckling, W.H. (1976). Theory of the Firm; anagerial Behavior, Agency Costs, and Ownership Structure. Journal of Financial Economics, No. 3 (4), pp. 305-360.
5. Milgrom, P. \& Roberts, J. (1993). Economia de la organización y gestión de empresa. Barcelona: Editorial Ariel Economía.

6. Pérez, P. (1999). Retribución de altos directivos en empresas españolas: un enfoque de agencia. Tesis doctoral. Universidad de Valladolid, Valladolid, España.

7. Robertson, M. \& O’Malley, G. (2000). Knowledge Management Practices within a KnowledgeIntensive Firm: the Significance of the People Management Dimension. Journal European Industrial of Manpower, No. 24 (1), pp. 241-253.

8. SAA, P.y García, J. (2000). El valor estratégico de los recursos humanos según la visión de la empresa basada en los recursos. Revista Europea de Dirección y Economia de la Empresa, Núm. 9 (2), pp. 97-116.

9. Vera, D.y Crossan, M.(2003). Organizational learning and knowledge management towards and integrative framework. The Blackwell Handbook of Organizational Learning and Knowledge Management, pp. 122-142.

10. Ulrich, D. \& Smallwood, N. (2005). HR's new RoI: Return on Intangibles. Human Resource Management. No. 44 (2), pp. 137-142. 